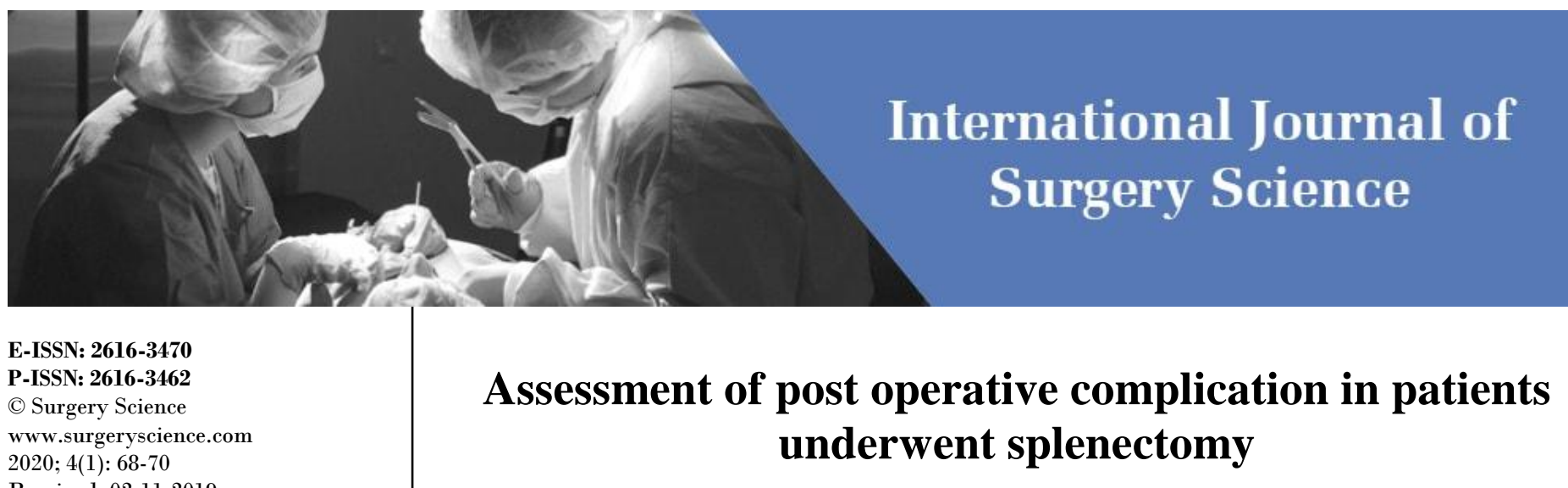

Received: 02-11-2019

Accepted: 05-12-2019

Nikhil Singh

Associate professor, Department of General Surgery Government

Medical College Kannauj, Uttar Pradesh, India

Corresponding Author: Nikhil Singh

Associate professor, Department of

General Surgery Government

Medical College Kannauj, Uttar

Pradesh, India

\section{Nikhil Singh}

DOI: https://doi.org/10.33545/surgery.2020.v4.i1a.310

\section{Abstract}

Background: Splenectomy is usually performed for patients with severe splenic trauma. The present study assessed complication in patients of splenectomy.

Materials \& Methods: The present study was conducted on 125 patients of both genders who underwent splenectomy. In all cases laparoscopic splenectomy was performed as per standardized operating procedure. Post operative complications were recorded

Results: Out of 125 patients, males were 70 and females were 55. The operative time was 134.2 minutes, transfusion was required in 45 patients and hospital stay was 4.2 days. Complications were hemoperitoneum in 2, pneumonia in 1 , wound sepsis in 6 , urinary infection in 2 , atelactasis in 1 , catheter sepsis in 2 and post operative ileus in 4 patients. The difference was significant $(p<0.05)$.

Conclusion: Authors found that common complications were hemoperitoneum, pneumonia, wound sepsis, urinary infection, atelactasis, catheter sepsis and post operative ileus.

\section{Keywords: atelactasis, splenectomy, pneumonia}

\section{Introduction}

Subtotal splenectomy was also performed in patients with Gaucher's disease in which giant splenomegaly caused abdominal discomfort, respiratory distress and walking difficulties. A total splenectomy in this disease frequently causes liver enlargement and bone fractures due to deposition of glucocerebroside. Lethal infection is another complication that may follow total splenectomy. To avoid this complication, partial splenectomy, preserving the splenic pedicle has been proposed.

Splenectomy is usually performed for patients with severe splenic trauma, portal hypertension, splenomegaly due to hematologic diseases, or splenic tumors. Intraperitoneal hemorrhage is a fatal complication following splenectomy. It is associated with surgical manipulation and hematologic coagulation. For prompt management of bleeding, it is important to make the diagnosis promptly by carefully observing the clinical manifestation, monitoring the blood hemoglobin level, and imaging with ultrasonography ${ }^{[4]}$. Laparoscopic splenectomy can be safely performed and have gained wide clinical practice today. It leads to decrease in complication related to trauma, access to magnified view of the opposite side and avoidance of manipulation of left side of diaphragm ${ }^{[5]}$.

According to published reports, the presence of splenomegaly in children and teenagers leads to retarded of growth and late sexual development, and therefore total splenectomy is frequently indicated. Thrombosis of the extrahepatic portal system following splenectomy occurs in a small portion of patients and is mostly an unpredictable event. The present study assessed complication in patients of splenectomy.

\section{Materials \& Methods}

The present study was conducted in the department of general surgery in 2016-2017. It comprised of 125 patients of both genders who underwent splenectomy. All were informed regarding the study. Ethical approval was obtained from institutional ethical committee prior to the study.

General information such as name, age, gender etc. was recorded. In all cases laparoscopic splenectomy was performed as per standardized operating procedure. Post-operative complications were recorded. Results thus obtained were subjected to statistical analysis. 
P value less than 0.05 was considered significant.

\section{Results}

Table I: Shows that out of 125 patients, males were 70 and females were 55 .

\begin{tabular}{|c|c|c|}
\hline \multicolumn{3}{|c|}{ Total- 125 } \\
\hline Gender & Males & Females \\
\hline Number & 70 & 55 \\
\hline
\end{tabular}

Table I shows that out of 125 patients, males were 70 and females were
55.

Table II: Assessment of parameters

\begin{tabular}{|c|c|}
\hline Parameters & Value \\
\hline Operative time (min) & 134.2 \\
\hline Transfusion & 45 \\
\hline Hospital stay (days) & 4.2 \\
\hline
\end{tabular}

Table II, graph I shows that operative time was 134.2 minutes, transfusion was required in 45 patients and hospital stay was 4.2 days.

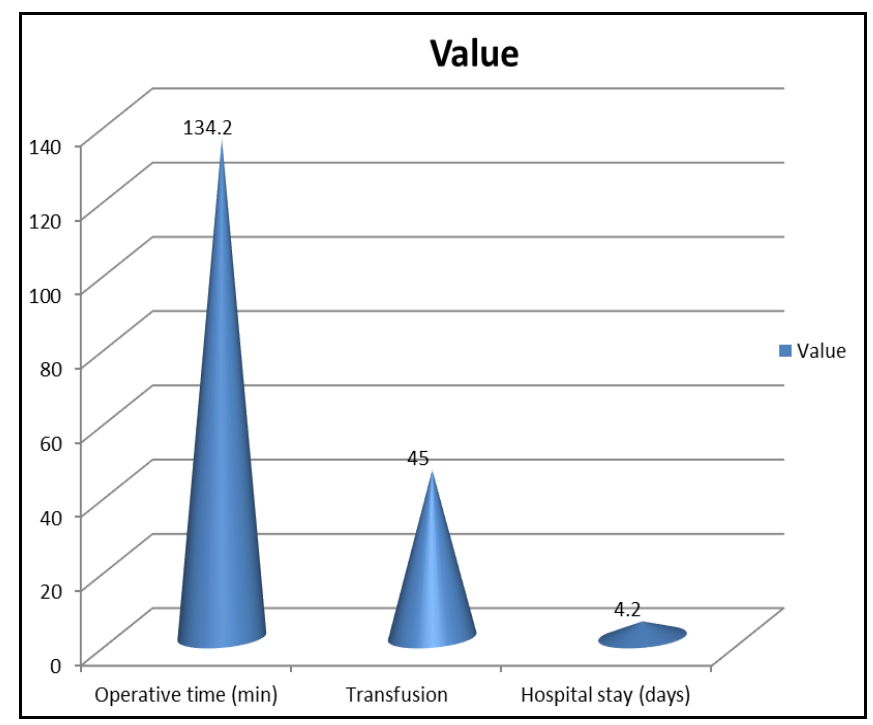

Graph I: Assessment of parameters

Table III: Complications in patients

\begin{tabular}{|c|c|c|}
\hline Complications & Number & P value \\
\hline Hemoperitoneum & 2 & \\
\hline Pneumonia & 1 & \multirow{2}{*}{0.01} \\
\hline Wound sepsis & 2 & \\
\hline Urinary infection & 1 & \\
\hline Atelactasis & 1 & \\
\hline Catheter sepsis & 2 & \\
\hline Post-operative ileus & 4 & \\
\hline
\end{tabular}

Table III, graph II shows that complications were hemoperitoneum in 2, pneumonia in 1, wound sepsis in 6, urinary infection in 2, atelactasis in 1, catheter sepsis in 2 and post operative ileus in 4 patients. The difference was significant $(p<0.05)$.

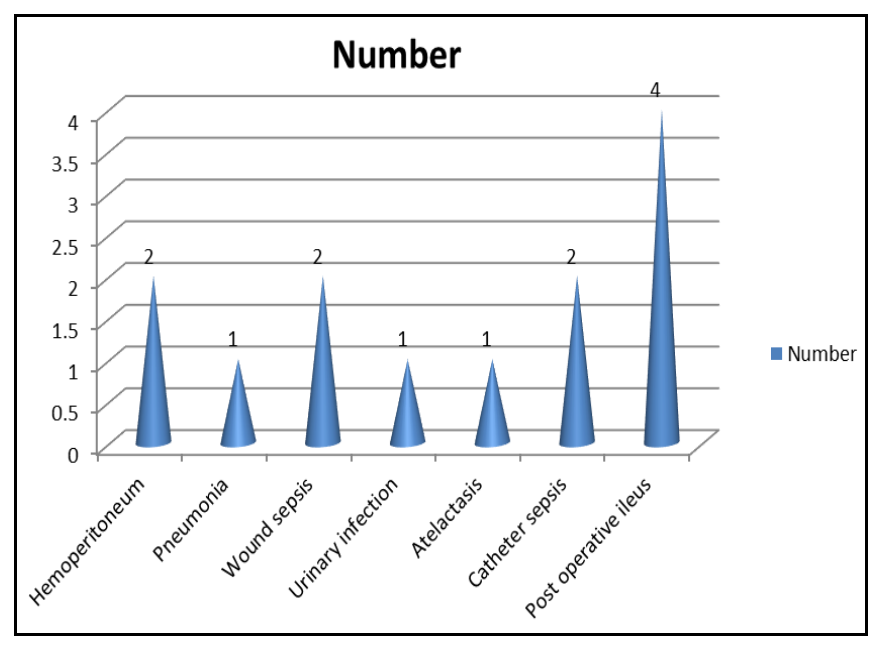

Graph I: Complications in patients

\section{Discussion}

Laparoscopic approach to diseases related to solid organ such as spleen and liver has lagged behind operations on hollow viscous because of problems related to hemostasis and extraction of specimen. It also entails difficulty because of frail nature of spleen and complex. Clinical assessment begins with a thorough history and examination ${ }^{[6]}$. The history may elicit symptoms of pressure effects from the enlarged spleen, such as left hypochondrial discomfort or early satiety ${ }^{[7]}$. The present study assessed complication in patients of splenectomy.

In present study, out of 125 patients, males were 70 and females were 55. The operative time was 134.2 minutes, transfusion was required in 45 patients and hospital stay was 4.2 days. Park et al. ${ }^{[8]}$ conducted a study in 86 patients who had undergone surgery. The surgical procedure was performed for one of the following conditions: portal hypertension due to schistosomiasis $(n=43)$, trauma $(\mathrm{n}=31)$, Gaucher's disease $(\mathrm{n}=4)$, myeloid hepatosplenomegaly due to myelofibrosis $(n=3)$, splenomegalic retarded growth and sexual development $(n=2)$, severe pain due to splenic ischemia $(n=2)$ and pancreatic cystadenoma $(n=1)$. Patients underwent a hematologic exam, an immunologic assessment, abdominal ultrasonography, computed tomography, scintigraphy and endoscopy. Increased white blood cell count and platelets were the only hematological abnormalities. No immunologic deficit was found. Esophageal varices were still present in patients who underwent surgery because of portal hyperension although without rebleeding. The ultrasound, tomography and scintigraphy exams confirmed the presence of functional splenic remnants without significant size alteration. We found that complications were hemoperitoneum in 2, pneumonia in 1 , wound sepsis in 6 , urinary infection in 2 , atelactasis in 1, catheter sepsis in 2 and post-operative ileus in 4 patients. 
Targarona et al. ${ }^{[9]}$ found that the laparoscopic splenectomy was successfully performed on all 86 patients. No operative complications, such as peritoneal cavity infection, massive bleeding after operation and adjacent organs injured were observed. There was no death related to the operation. The study showed that different operative positions could significantly influence the manipulation of LS. The right lateral position had more advantages than the lithotomic position and the right recumbent position in LS.

Morgenstern et al. ${ }^{[10]}$ considered 50 age- and gender-matched case controls undergoing OS without prior SAE during the same period (control group). Perioperative and clinical outcomes were compared between the two groups. No significant differences were found between the two groups for age, gender and laboratory investigations $(\mathrm{p} \geq 0.250)$. Mortality rate was zero in both groups. No patients of the study group needed perioperative blood transfusion in comparison with patients of the control group $(p=0.003)$. A significant increase in platelet count was noted in the study group after SAE compared to the control group $(p=0.024)$. No significant differences between the two groups were observed for operating time, postoperative complications and postoperative stay

\section{Conclusion}

Authors found that common complications were hemoperitoneum, pneumonia, wound sepsis, urinary infection, atelactasis, catheter sepsis and post-operative ileus.

\section{References}

1. Tulman S, Holcomb GW, Karamanoukian HL, Reynhout J. Laparoscopic splenectomy. J Pediatr Surg. 1993; 28:689-92.

2. Carroll BJ, Phillips EH, Semel CJ. Laparoscopic splenectomy. Surg Endosc. 1992; 6:183-5.

3. Flower JL, Lefor AT, Steers J. Laparoscopic splenectomy in patients with hematologic diseases. Ann Surg. 1996; 224(1):19-28.

4. Sotomayor-Ramires RK. Efficacy and safety of laparoscopic splenectomy. Bol Asoced PR. 2009; 101(2):43-9.

5. Petroianu A, Berindoague RN. Laparoscopic subtotal splenectomy. Minerva Chir. 2004; 59:501-505.

6. Govrin-Yehudain J, Bar-Maor JA. Partial splenectomy in Gaucher's disease. Israel Med Sci. 1980; 16:665-668.

7. Petroianu A, Petroianu LPG. A twelve-year-follow-up of subtotal splenectomy and central splenorenal shunt for treatment of schistosomatic portal hypertension. Folha Méd. 1998; 116:107-109

8. Park A, Marcaccio M, Sternbach M, Witzke D, Fitzgerald P. Laparoscopic vs. open splenectomy. Arch Surg. 1999; 134:1263-9.

9. Targarona EM, Espert JJ, Cerdan G, Balagué C, Piulachs J, Sugrañes G et al. Effect of spleen size on spelenectomy outcome. A comparison of open and laparoscopic surgery. Surg Endosc. 1999; 13:559-62.

10. Morgenstern L, Phillips EH, Fermelia D et al. Near-total splenectomy for massive splenomegaly due to Gaucher disease. Mount Sinai J Med. 1986; 53:501-505. 